\title{
Dispositivos de monitoramento: aliados ou inimigos? Construindo um protocolo de limpeza/desinfecção para enfermagem*
}

\section{Monitoring devices: allies or enemies? Building a protocol of cleaning / disinfection for nursing}

Roberta Pereira Spala Neves ${ }^{1}$ - Fátima Helena do Espirito Santo ${ }^{2}$

\begin{abstract}
RESUMO
Este estudo aborda a limpeza e desinfecção dos principais dispositivos não-críticos de monitoração, considerando sua participação na transmissão das infecções relativas aos cuidados médicos. 0 objetivo deste estudo é descrever o desenvolvimento de um protocolo de limpeza e desinfecção dos principais dispositivos não-críticos utilizados na monitorização, baseado em melhores práticas, evidenciadas na literatura. É um estudo descritivo e exploratório, guiado pela pesquisa baseada em evidência, conduzida de Março de 2012 à Julho de 2014, explorando as seguintes fases: uma revisão integrativa de literatura; preparação de protocolo de desinfecção e limpeza de dispositivos nãocríticos de monitoramento e avaliação por experts. Entre as boas práticas encontradas na literatura sobre limpeza e desinfecção destes dispositivos destaca-se o uso do álcool etílico à $70 \%$ como desinfetante principal da escolha da necessidade, utilizado em intervalos regulares. 0 protocolo foi avaliado por um painel de peritos, incluindo nesta avaliação a possibilidade de aplicação no contexto dos cuidados médicos nas instituições de saúde. Acredita-se que este protocolo possa contribuir como guia das práticas profissionais de saúde, minimizando os riscos de infecções no cotidiano do serviço de saúde.
\end{abstract}

Palavras-chave: Termômetros; Esfignomômetros; Eletrocardiografia; Oximetria; Infecção Hospitalar.

\begin{abstract}
This study approaches the cleaning and disinfection of the main non-critical monitoring devices, considering their participation in the transmission of infections related to health care and the goal this study is describe the development of a cleaning protocol and disinfection of the main non-critical monitoring devices based on best practices and evidence from the literature. It is a descriptive and exploratory study, guided by evidence-based research, conducted from March 2012 to July 2014 in the following stages: an integrative literature review, preparation of non-critical cleaning protocol and disinfection monitoring devices and evaluation expertise. Among the good practices identified in the literature on cleaning and disinfection of these devices include the use of ethanol to $70 \%$ as the main disinfectant of choice and the need for cleaning and disinfection of these devices at regular intervals, set the protocol that was assessed by the experts as appropriate panel with application possibility in the healthcare context of health institutions. We believe that this protocol may contribute to guide health professional's practices minimize the risk of infection associated with their use in everyday health services.
\end{abstract}

Keywords: Thermometers; Sphygmomanometers; Electrocardiography; Oximetry; Hospital Infection.

${ }^{1}$ Roberta Pereira Spala Neves. Enfermeira, Mestre em Enfermagem, Mestrado Profissional em Enfermagem Assistencial, Escola de Enfermagem Aurora de Afonso Costa, Universidade Federal Fluminense/EEAAC/UFF. $1^{\circ}$ Tenente Enfermeira do Corpo de Bombeiros do Estado do Rio de Janeiro. Rio de Janeiro (RJ), Brasil

E-mail: roberta.spala@yahoo.com.br.

${ }^{2}$ Fátima Helena do Espírito Santo. Enfermeira, Doutora em Enfermagem, Professora Associada do Departamento de Enfermagem Médico-Cirúrgica, Escola de Enfermagem Aurora de Afonso Costa, da Universidade Federal Fluminense/EEAAC/UFF. Niterói (RJ), Brasil. E-mail: professorafh@vm.uff.br

*Artigo baseado em dissertação de Mestrado Profissional em Enfermagem Assistencial: Neves, RPS . Dispositivos de monitoramento não críticos: aliados ou inimigos? Construindo um protocolo de limpeza/desinfecção para enfermagem[dissertação]. Niterói: Escola de Enfermagem Aurora de Afonso Costa/Universidade Federal Fluminense; 2014. 


\section{INTRODUÇÃO}

O ambiente nos serviços de saúde tem sido foco de especial atenção para minimizar a disseminação de microorganismos, pois pode atuar como fonte de recuperação de bactérias multirresistentes causadoras das infecções relacionadas à assistência a saúde (IRAS). ${ }^{1}$

Nesse contexto, este estudo aborda os processos de limpeza e desinfecção dos dispositivos de monitoramento não críticos, são eles: termômetros, esfigmomanômetros, cabos de eletrocardiograma (ECG) e oxímetro, considerando a sua participação na transmissão das infecções relacionadas à assistência à saúde.

Apesar de serem considerados materiais de baixo risco para transmissão de infecções, uma vez que são artigos não críticos, cujo contato é restrito a pele íntegra do paciente ${ }^{2}$, alguns estudos têm apontado os dispositivos de monitoramento não críticos como uma das principais fontes de infecção por bactérias multirresistentes causadoras de surtos em ambiente hospitalar, principalmente em unidades de terapia intensiva, adulto ou neonatal.

Estudo realizado em 2012 identificou os sensores de oxímetro como principal reservatório causador do surto de Staphylococcus Aureus, com resistência heterogênea a glicopeptídeos, em uma unidade de terapia intensiva ${ }^{3}$. Outro estudo realizado em 2002 apontou os termômetros como uma das fontes causadoras de surto de Enterobacter cloacae em uma unidade neonatal, sugerindo assim a modificação do método de desinfecção deste dispositivo. Já estudo realizado em 2010 descreveu que, após a cultura de 320 cabos de ECG limpos, foi constatado crescimento bacteriano em 201 cabos, destes, foram identificadas nove espécies de risco, cinco espécies com risco potencial e 10 sem risco ou com risco raro 4 .

Bactérias patogênicas também foram detectadas nos esfigmomanômetros em estudo realizado por meio de cultura de 24 manguitos de pressão não invasiva, sendo constatado crescimento de microorganismo viável em todos os manguitos, dos quais 11 foram isolados organismos patogênicos tais como MSSA (Staphylococcus Aureos sensível a meticilina), MRSA (Stphylococcus Aureos resistente a meticilina) e Clostridium Difficile (C. Difficile) ${ }^{5}$.

Algumas bactérias como Staphylococcus Aureus resistentes à Meticilina e Enterococos à Vancomicina, são capazes de sobreviver dias, ou até semanas nas superfícies de ambientes de serviços de saúde ${ }^{6}$ Assim, é fundamental o aperfeiçoamento da limpeza e desinfecção desses dispositivos para eliminar esta via de contaminação ${ }^{1 .}$

Os estudos identificados sobre a temática apontam estes dispositivos como possíveis reservatórios de microorganismos patogênicos, necessitando rever os procedimentos de limpeza e desinfecção dos mesmos.

Assim, este estudo traz as recomendações de limpeza e desinfecção de cada um destes dispositivos de monitoramento não críticos, mencionados anteriormente, baseado nas boas práticas e conhecimentos encontrados na literatura, seguida de avaliação por um painel de experts em infecção hospitalar.
No que se refere às boas práticas, são concebidas como um "conjunto de procedimentos necessários para garantir a qualidade sanitária dos produtos e dos serviços em um processo de trabalho".

Portanto, a limpeza e desinfecção dos objetos inanimados é uma medida de controle fundamental das IRAS, interferindo diretamente em uma das cadeias de transmissão dos microrganismos. No entanto, para ser realizada efetivamente é preciso que a equipe de saúde esteja sensível ao problema a fim de aderir às medidas de controle implementadas.

Propor medidas eficazes para limpeza e desinfecção de equipamentos de monitoramento não críticos, como prevenção e controle de infecções, não garante que os pacientes, de um modo geral, estejam totalmente protegidos. Porém, a padronização e a sistematização do processo poderão contribuir para minimizar os riscos relacionados ao seu uso, contribuindo, com a redução dos índices de IRAS, das taxas de mortalidade e morbidade entre os pacientes internados, do tempo de internação e gastos hospitalares com este evento adverso.

Para isso é fundamental o treinamento dos profissionais, ampliação das pesquisas sobre o tema e divulgação da cultura da segurança do paciente visando reduzir ao máximo possível a ocorrência de eventos adversos e práticas inseguras que ameaçam a saúde dos pacientes. ${ }^{8}$

Assim essa pesquisa se justifica pela relação das IRAS e os dispositivos de monitoramento não críticos apontados na literatura e a necessidade de padronização da limpeza e desinfecção dos dispositivos não críticos nos serviços de saúde visando promover mais segurança tanto aos usuários quanto aos profissionais de saúde.

Para nortear o estudo elaborou-se a seguinte questão de pesquisa: Como deve ser realizada a limpeza e desinfecção dos principais dispositivos de monitoramento não críticos, a fim de reduzir seu potencial como veículo de infecção?

Sendo assim, o objetivo desta pesquisa é descrever a elaboração de um protocolo de limpeza e desinfecção dos principais dispositivos de monitoramento não críticos, com base nas boas práticas e evidências da literatura.

\section{MÉTODO}

Estudo descritivo, exploratório, com ênfase nos princípios da pesquisa baseada em evidências (PBE), no qual os problemas encontrados na prática são discutidos, à luz dos conhecimentos científicos 9 .

O estudo foi desenvolvido no período de março de 2012 a julho de 2014 seguindo as seguintes etapas: revisão integrativa da literatura, para identificação das boas práticas evidenciadas na literatura, elaboração do protocolo de limpeza e desinfecção de dispositivos de monitoramento não críticos e avaliação por expertises de enfermagem na área de infecção hospitalar. 


\section{Primeira Etapa: Revisão integrativa da literatura: buscando as boas práticas}

A revisão integrativa foi realizada cumprindo cada fase a seguir: identificação do tema, estabelecimento de critérios de inclusão e exclusão/busca na literatura, categorização dos estudos, análise crítica, discussão dos resultados e síntese do conhecimento ${ }^{10-11}$.

Para essa revisão foi definida como questão norteadora: Quais são as evidências científicas sobre as IRAS associadas aos principais dispositivos de monitorização não invasivo ?

A busca na literatura foi realizada pelo pesquisador nas principais bases de dados on line: LILACS, IBECS, MEDLINE e SCIELO mediante os seguintes critérios de inclusão: artigos científicos disponíveis na integra online nos idiomas inglês, espanhol e português, publicadas nos últimos 16 anos. Inicialmente foram definidos artigos publicados nos últimos 10 anos a fim de selecionar estudos mais recentes sobre a temática, no entanto apenas sete artigos foram encontrados e, dentre estes, nem todos os dispositivos deste estudo foram contemplados. Assim para alcançar mais estudos que pudessem contribuir com esta pesquisa trazendo dados importantes, o período de busca foi ampliado para 16 anos, desta forma foram incluídos mais seis artigos contemplando todos os dispositivos.

Foram critérios de exclusão: estudos tipo carta, editorial, opiniões, normativas e resoluções e estudos que não apresentavam relação com a temática.

O período de busca nas bases de dados foi entre os meses de julho a setembro de 2013, com associação do descritor cross infection com os seguintes descritores: oximetry, electrocardiography, sphygmomanometers e thermometers. No total foram encontrados 252 artigos, porém apenas 33 tinham relação com a temática e, desses, 16 tinham sua publicação há mais de 16 anos, dos quais quatro não estavam disponíveis na íntegra, obtendo-se 13 artigos que compõem esta revisão.

Assim, no total foram selecionados para o estudo 13 artigos que atenderam aos critérios de inclusão e exclusão. Esses artigos foram lidos na integra e, seguindo as fases da revisão integrativa, foi realizada a categorização, análise, discussão e síntese do conhecimento científico sobre o tema.

\section{Segunda etapa: Construindo o protocolo}

0 protocolo é um instrumento que contribui com a modificação do comportamento dos profissionais de saúde, tendo como foco a padronização de condutas, este foi o documento de escolha para padronizar o processo de limpeza e desinfecção dos principais dispositivos de monitoramento não críticos $^{12 .}$ Sua construção compreendeu o período de outubro a dezembro de 2013 e para definir as recomendações do protocolo foram utilizadas as boas práticas identificadas na revisão integrativa, além do conhecimento adquirido em livros, teses dissertações e documentos nacionais e internacionais.

\section{Terceira etapa: Validação do protocolo por experts na área de infecção hospitalar.}

Esta etapa consistiu na avaliação do protocolo por um painel de experts em Infecção hospitalar que implicou na elaboração de critérios de seleção e exclusão dos experts e de um roteiro de avaliação a ser utilizado pelos mesmos.

Para elaboração dos critérios de seleção de expert na área de infecção hospitalar, foi feita uma adaptação do modelo sugerido por Fehring em 1994, que utiliza critérios para seleção de expert na área de diagnóstico de enfermagem ${ }^{13}$. Assim, adaptando o modelo sugerido por Fehring (1994), e mantendo a pontuação mínima de cinco pontos para considerar o profissional expert em infecção hospitalar, os critérios e pontuações utilizados neste estudo são mostrados no quadro 1.

Quadro 1: Critérios de seleção de experts na área de infecção hospitalar: adaptação do modelo sugerido por Fehring

\begin{tabular}{|l|c|}
\hline $\begin{array}{l}\text { Doutorado com tese defendida na área } \\
\text { de Infecção Hospitalar; }\end{array}$ & 4 \\
\hline $\begin{array}{l}\text { Mestrado com dissertação defendida } \\
\text { na área de Infecção Hospitalar; }\end{array}$ & 3 \\
\hline $\begin{array}{l}\text { Pós-graduação lato sensu em infecção } \\
\text { hospitalar; }\end{array}$ & 1 \\
\hline $\begin{array}{l}\text { Produção cientifica sobre infecção } \\
\text { hospitalar nos últimos } 10 \text { anos }\end{array}$ & $\begin{array}{c}1 \text { ponto para cada } \\
\text { produção científica }\end{array}$ \\
\hline $\begin{array}{l}\text { Experiência de pelo menos } 1 \text { ano na } \\
\text { CCIH nos últimos } 10 \text { anos. A cada ano } \\
\text { o profissional receberá } 1 \text { ponto. }\end{array}$ & 1 ponto a cada ano \\
\hline
\end{tabular}

Fonte: Adaptado de Critério de seleção de experts na área de infecção hospitalar na dissertação de Neves, $2014^{14}$.

Quanto ao roteiro de avaliação do protocolo para o painel de experts, este foi elaborado uma lista de itens em que eles avaliavam cada recomendação, utilizando uma escala do tipo Likert de quatro pontos de suporte, onde o expert poderia analisar cada item como inadequado $=0$ pontos, menor parte adequado $=0,33$, maior parte adequado $=0,67$ e totalmente adequado $=1$ ponto, quando não consideravam a recomendação totalmente adequada, havia um espaço para descrição de alguma observação, apontando o motivo de sua discordância ou pra sugerir uma nova recomendação.

Para a busca dos experts foi utilizada a técnica bolade-neve que é uma forma de amostra não probabilística, onde os participantes iniciais indicam novos participantes e, assim, sucessivamente, até que seja alcançado o ponto de saturação ${ }^{15}$.

O contato com os profissionais ocorreu via correio eletrônico. Todo esse processo, que compreendeu a busca e contato com os expert, até o recebimento dos questionários respondidos ocorreu no período de maio a julho de 2014, totalizando cinco experts neste estudo. Todos os experts retornaram suas avaliações dentro do período proposto (7dias) e não foi necessária nova rodada de avaliação pelo painel de experts. 


\section{Análise das respostas}

Encerrada a etapa de avaliação, foi calculada a média ponderada das respostas, conforme proposto por Ferhing, para se obter o grau de concordância entre os experts ${ }^{13}$.

Para análise do grau de concordância entre os mesmos foi considerada a pontuação de cada item da escala do tipo Likert sendo definido que as recomendações que apresentassem um grau de concordância maior que 0,8 não precisariam sofrer alterações, logo, seriam mantidas no protocolo. Assim, as recomendações que alcançaram um grau de concordância maior que 0,5 e menor ou igual 0,8poderiam, ou não, sofrer alterações, considerando a análise das observações. Porém, as recomendações que obtivessem média menor ou igual 0,5 - seriam, necessariamente, revisadas ou descartadas.

Após análise das respostas procedeu-se elaboração do protocolo final que pretende contribuir com a diminuição da colonização dos principais equipamentos de monitoramento não críticos, a fim de prevenir as IRAS transmitidas a partir destes reservatórios.

\section{Aspectos éticos}

O projeto de pesquisa foi aprovado pelo Comitê de Ética em Pesquisa do Hospital Universitário Antônio Pedro/ Universidade Federal Fluminense (UFF), sob parece $n^{\circ}$ 499.444. Os experts foram esclarecidos sobre a natureza da pesquisa, seus objetivos e procedimentos e quanto à assinatura do Termo de Consentimento Livre e Esclarecido (TCLE), conforme preconizado na Resolução 466/12/2012 do Conselho Nacional de Saúde.

\section{RESULTADOS}

Como resultado desta pesquisa obteve-se o protocolo de limpeza e desinfecção dos principais dispositivos de monitoramento não críticos, antes e, após, avaliação por um painel de experts em infecção hospitalar.

Nesse artigo são apresentados os processos de limpeza e desinfecção de cada dispositivo estudado que deu origem ao protocolo proposto.

Esses procedimentos foram inicialmente descritos com base na revisão integrativa da literatura, complementado com livros, manuais, guidelines, dissertações e teses para elaboração das recomendações do protocolo proposto. Após avaliação do painel de experts, foram consideradas as sugestões de alterações e aperfeiçoamentos de cada processo para as recomendações descritas no protocolo final.

A seguir são apresentados na forma de diagrama os procedimentos de limpeza e desinfecção após a avaliação pelos experts.

Para as braçadeiras de pano serão apresentadas duas opções de procedimento. A primeira opção utiliza-se detergente desinfetante a base de quaternário de amônia, e a segunda opção lavagem térmica.

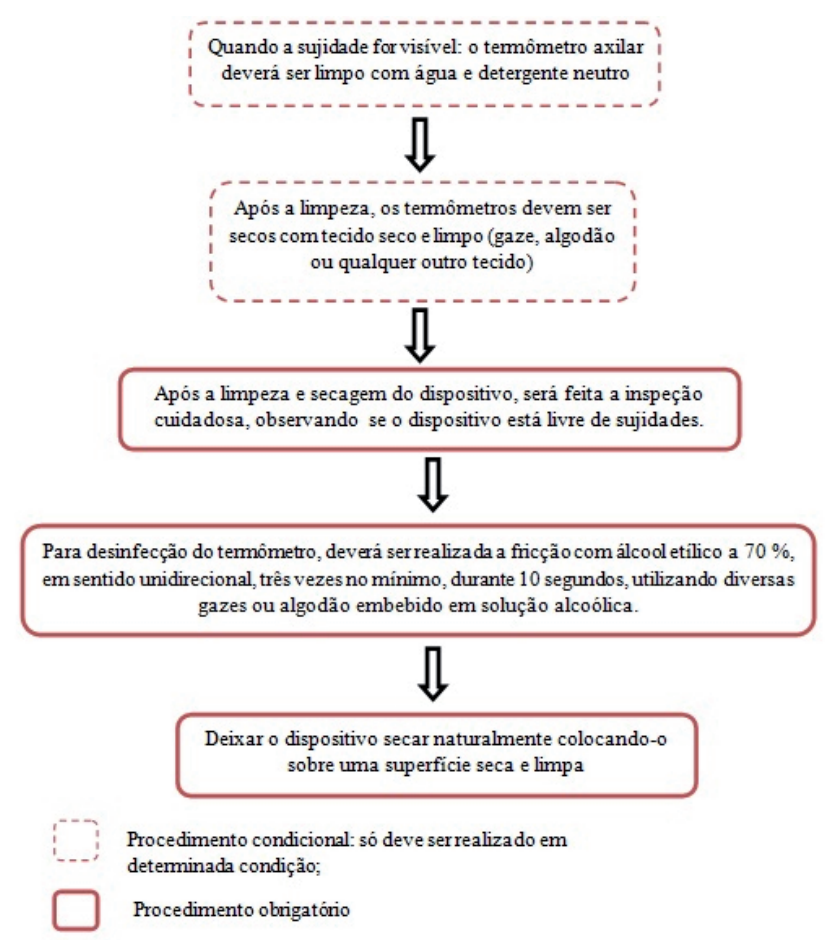

Figura 1- Processo de limpeza e desinfecção dos termômetros axilares Fonte: Diagrama do processo de limpeza e desinfecção dos termômetros axilares sugeridos na dissertação (NEVES, 2014) ${ }^{14}$.

Desconectar o manguito das demais partes do esfigmomanômetro.

\section{$\sqrt{ }$}

- Quando posaível, retirar o manguito de dentro da braçadeira - Senäo for possível retirar o manguito, proteger as entradas dos tubos de borracha que conectam o manguito a outras pertes do dispositivo e permitem acesso a parte intema dos manguitos.

\section{$\sqrt{n}$}

Realizar a limpeza através da friç̧ão com água e detergente neutro;

\section{$\sqrt{1}$}

Após a friç̧ão deve ser enxaguado abundantemente

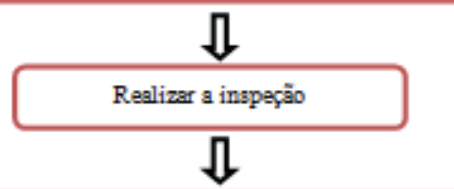

Secar com a ajuda de panos secos ou ar comprimido

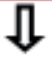

Realizar a desinfeç̧ão através da friç̧ão com álcool etílico a $70 \%$ por no mínimo três veres em sentido unidirecional, durante 10 segundos;

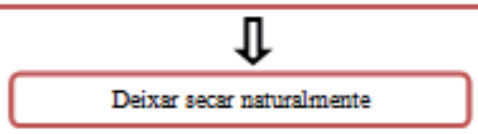

Procedimento obrigatório

Figura 2- Processo de limpeza e desinfecção das braçadeiras de nylon do esfigmomanômetro

Fonte: Diagrama do processo de limpeza e desinfecção das braçadeiras de nylon do esfigmomanômetro sugeridos na dissertação de Neves, $2014^{14 .}$ 


\section{1a opção:}

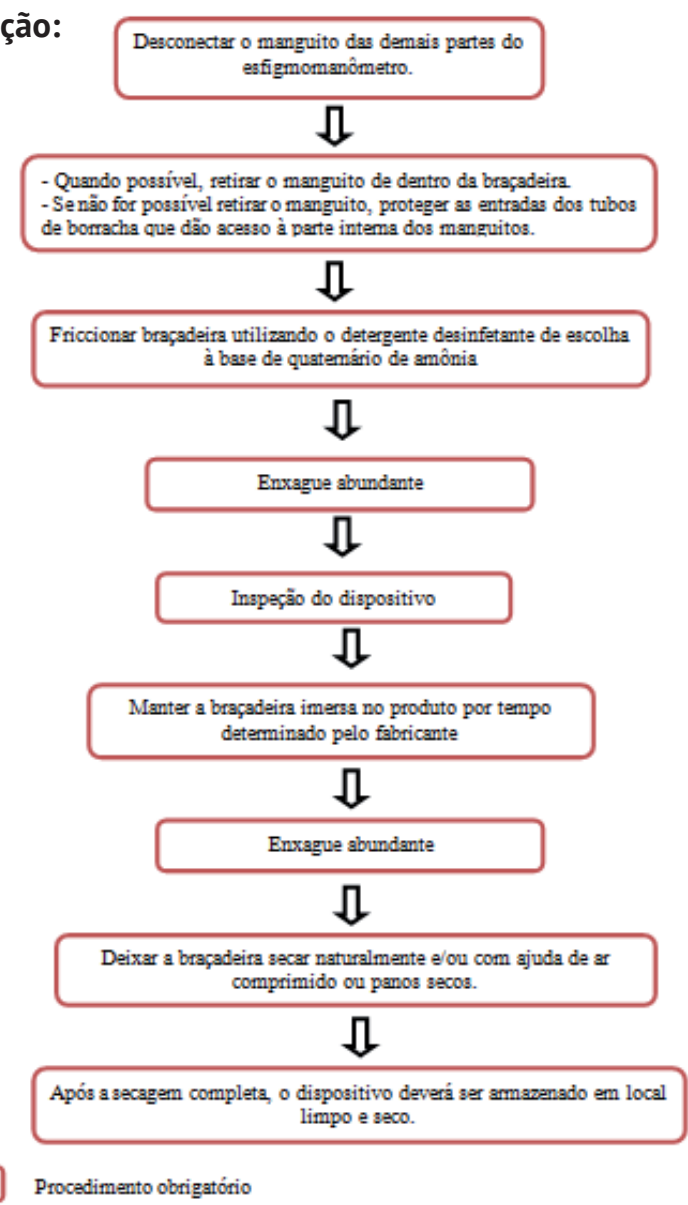

Figura 3- Processo de limpeza e desinfecção das braçadeiras de pano dos esfignomanômetro

Fonte: Diagrama da $1^{\text {a }}$ opção do processo de limpeza e desinfecção das braçadeiras de pano dos esfignomanômetro sugeridos na dissertação de Neves, 20144.

\section{2a opção:}
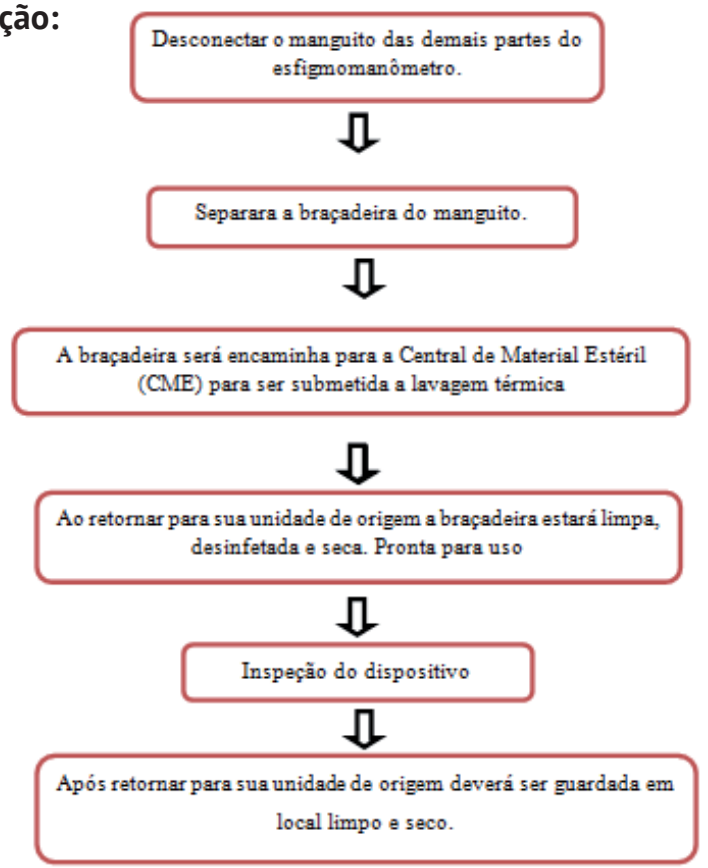

Procedimento obrigatório

Figura 4 - Processo de limpeza e desinfecção das braçadeiras de pano dos esfignomanômetro

Fonte: Diagrama da $2^{\mathrm{a}}$ opção do processo de limpeza e desinfecção das braçadeiras de pano dos esfignomanômetro sugeridos na dissertação de Neves, 2014 ${ }^{14}$.

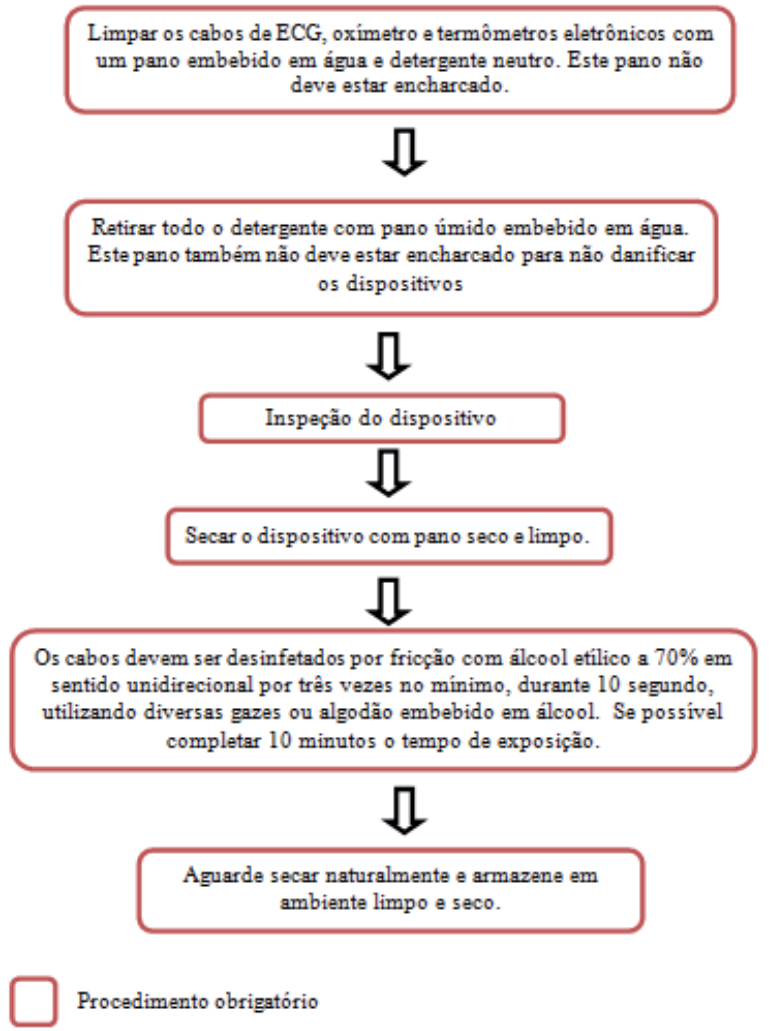

Figura 5 - Processo de limpeza e desinfecção dos cabos de ECG e oxímetro

Fonte: Diagrama do processo de limpeza e desinfecção dos cabos de ECG e oxímetro sugeridos na dissertação (NEVES, 2014) ${ }^{14}$

Após serem definidos todos os procedimentos descritos anteriormente estes foram colocados em formato de protocolos. 0 protocolo traz a sequência dos passos a serem seguidos para executar uma tarefa, e podem ser elaborados sob a forma de texto e diagramas, que vão facilitar a compreensão do processo de trabalho por parte dos profissionais ${ }^{16 .}$ Desta forma, para elaboração do protocolo de limpeza e desinfecção dos principais dispositivos de monitoramento não críticos, foi utilizado um modelo de instrumento normativo, construído pela Assessoria de Qualidade da Secretaria Estadual de Saúde, no ano de $2000^{17}$.

\section{DISCUSSÃO}

Após revisão integrativa da literatura, foi realizada leitura minuciosa de cada estudo e feita análise crítica que permitiu a discussão dos resultados, onde o autor compara os dados evidenciados nos estudos com o conhecimento teórico para gerar cada recomendação nos processos de limpeza e desinfecção dos dispositivos em questão. Concomitante as recomendações, foram apresentadas, na pesquisa original, a síntese do conteúdo que são as boas práticas, identificadas nos estudos para a limpeza e desinfecção dos dispositivos não críticos estudados nesta pesquisa.

A fim de obter o protocolo final, as recomendações foram analisadas pelos experts, os quais atribuíram uma 
pontuação quanto à concordância ou discordância de cada recomendação e sugeriram alterações ou até mesmo descarte da recomendação.

As recomendações foram divididas em recomendações gerais, recomendações para cada um dos dispositivos.

\section{Recomendações gerais:}

1: Lavar as mãos, sempre, antes e depois de qualquer procedimento.

- Obteve um grau de concordância igual a 1, desta forma, se mantém a mesma para o protocolo final.

2: Após a limpeza/ desinfecção dos equipamentos deve ser realizada inspeção.

- A segunda recomendação sofrerá uma pequena alteração, conforme sugestão de um dos experts, que orienta que a inspeção seja realizada após a limpeza, e não, após a desinfecção.

Nova recomendação número 2, após analise dos experts: após a limpeza, os dispositivos devem ser inspecionados para então serem desinfetados.

\section{Recomendações para limpeza/desinfecção termômetros axilares de mercúrio:}

3: Quando o dispositivo estiver sujo visivelmente, deverá ser limpo com água e detergente neutro, realizando a fricção do saneante no dispositivo, através de uma gaze ou qualquer tecido não abrasivo;

4: Após a limpeza realizar o enxágüe;

5: Após o enxágüe realizar a secagem;

- As recomendações 3,4 e 5 com grau de concordância $=1-$ sem alterações

6: Realizar fricção com álcool etílico a $70 \%$, por 10 segundos, utilizando diversas compressas de gases e algodão embebido na solução alcoólica.

- Com uma média ponderada de 0,93, a recomendação não precisaria sofrer alteração, porém, um dos experts trouxe uma contribuição que tornou a recomendação mais completa. Sendo assim, segue a nova recomendação.

Nova recomendação número 6: realizar fricção com álcool etílico a 70 \%, no sentido unidirecional, três vezes no mínimo, durante 10 segundos, utilizando diversas compressas de gases e algodão embebidos na solução alcoólica.

7: Procedimento não obrigatório: se for possível, manter o equipamento submerso por 10 minutos em álcool etílico a $70 \%$, e por fim, descartar essa solução.

- Essa recomendação foi descartada, não pelo grau de concordância, que apesar de ter sido baixo,ultrapassou 0,5 de média ponderal, mas pela justificativa dos experts que afirmaram que a imersão em álcool etílico não terá efeito para desinfetar o dispositivo sem a fricção, pois, o álcool é volátil e necessita da ação mecânica para promover a desinfecção.

8: Deixar secar naturalmente.

- Não sofreu alteração obtendo uma média ponderada de 0,86.

9: 0 termômetro axilar deve ser limpo e desinfetado a cada procedimento realizado.

- Com uma média ponderada de 1 , a recomendação segue a mesma.

\section{Recomendação para limpeza/desinfecção da braçadeira do esfigmomanômetro}

10: 0 dispositivo deve ser limpo com um detergente desinfetante a base de quaternário de amônia.

11: Primeiro será feita a limpeza do equipamento através da fricção com o detergente desinfetante a base de quaternário de amônia.

12: Após a fricção o dispositivo deverá ser enxaguado abundantemente.

13: Deverá ser retirada a água bruta com uma secagem superficial.

14: Manter o dispositivo submerso em detergente desinfetante a base de quaternário de amônia pelo tempo de exposição determinado pelo fabricante.

15: Ao término do tempo de exposição, o dispositivo deverá ser enxaguado abundantemente

16: Depois realizar a secagem completa naturalmente ou com a ajuda de ar comprimido ou/e panos secos.

- As recomendações de número 10 a 16 estão todas relacionadas à limpeza e desinfecção das braçadeiras de pressão não invasiva, todos os itens obtiveram um grau de concordância igual, ou menor que 0,80. Assim sendo, serão consideradas as sugestões e observações dos experts, a fim de produzir novas recomendações e até outras formas de realizar esta limpeza/desinfecção.

Inicialmente, um dos experts chama atenção para desconectar as partes do esfigmomanômetro antes de realizar a limpeza e desinfecção, uma vez que, cada parte é composta por um material diferente. Desta forma, é importante conhecer cada uma dessas partes.

Assim, surgem as seguintes recomendações:

- Antes de iniciar a limpeza e desinfecção das braçadeiras, os manguitos devem ser desconectados das demais partes;

- Retirando o manguito de dentro da braçadeira e, caso seja observado sujidade no manguito, este deverá ser limpo com água e detergente neutro, protegendo as entradas dos tubos de borracha que dão acesso a parte interna dos manguitos, em seguida, deverão ser enxaguados e secos;

- Após a limpeza, ou na ausência de sujidades, ele deverá ser desinfetado com álcool etílico a $70 \%$, através da 
fricção, por no mínimo três vezes, em sentido unidirecional, durante 10 segundos, utilizando diversas compressas de gases ou algodão embebidos nesta solução alcoólica;

Os experts, também, sugeriram que fosse especificado qual é o tipo de material da braçadeira, pois, existem braçadeiras de pano e de nylon e, para cada material terá uma orientação específica. Desta forma, no protocolo as orientações serão diferenciadas para as braçadeiras, de acordo com o seu material.

Desta forma de acordo com a orientação dos experts surgem as seguintes recomendações, já descritas no diagrama apresentado nos resultados.

Para a limpeza e desinfecção da braçadeira de pano são descritas duas opções, estas também estão descritas nos diagramas apresentados nos resultados.

O uso do quartenário de amônio como opção de desinfetante para braçadeiras de esfigmomanômetro foi contestado por um dos experts, alegando que este produto é, normalmente, utilizado para desinfecção de superfícies e equipamentos da área de alimentação, no entanto, foram encontrados referências e estudos que mostram bons resultados deste produto como desinfetante deste equipamento. Assim sendo manteve-se como opção de desinfetante para este equipamento.

A segunda opção que surge entre as sugestões dos experts para limpeza/desinfecção das braçadeiras de pano é a lavagem térmica. No entanto, o uso da lavagem térmica como uma opção para limpar e desinfetar as braçadeira do esfigmomanômetro apresenta algumas limitações, pois, é necessário que a unidade de saúde possua este equipamento, para então, realizar a lavagem térmica. A sergunda limitação está no próprio dispositivo de saúde, pois, o manguito não suporta a lavagem térmica. Desta forma, se for utilizado o modelo de esfigmomanômetro onde a braçadeira está costurada e não permite o acesso ao manguito, este processo não pode ser realizado.

17: Manguito utilizado em CTI: realizar a limpeza do dispositivo, se estiver visivelmente sujo, a cada mudança de turno e após a alta do paciente.

18: Manguitos utilizados em enfermaria de forma intermitente (uso coletivo): realizar a limpeza do dispositivo se estiver visivelmente sujo, a cada mudança de turno e após o contato com pacientes colonizados/infectados por bactérias multirresistentes.

19: Manguitos utilizados em sala cirúrgica: Realizar a limpeza, quando visivelmente sujo, após o contato com pacientes colonizados/infectados por bactérias multirresistentes e, após a última cirurgia do dia.

20: Manguitos utilizados em ambulatório: realizar a limpeza, se visivelmente sujo, uma vez por mês e após contato com pacientes colonizados/infectados por bactérias multirresistentes.

Inicialmente a frequência da limpeza e desinfecção das braçadeiras foi definida de acordo com o local de uso, no entanto com as sugestões dos experts a limpeza também foi diferenciada de acordo com o tipo de material da braçadeira.

Quando utilizado braçadeira de nylon este material permite desinfecção através da fricção com álcool etílico a $70 \%$, sendo este um procedimento mais rápido e prático, assim as unidades de saúde devem dar preferência ao uso deste tipo de material, o que permite uma desinfecção rápida na ausência de sujidades visíveis.

Um dos expert também sugeriu baseado no Manual de Investigação e Controle de bactérias multirresistentes de $2007^{(18)}$, que um tecido limpo e fino seja utilizado entre a pele do paciente e a braçadeira, evitando, o contato direto, visto a dificuldade em realizar a limpeza e desinfecção deste dispositivo.

Assim, as recomendações de 17 a 20 não sofreram alterações, sendo acrescidos os seguintes cuidados: Dar preferência às braçadeiras de nylon e, nesse caso, realizar a desinfecção com álcool etílico a $70 \%$, entre o uso em pacientes distintos. Também, deve ser utilizado um pano limpo e fino para diminuir o contato entre a pele do paciente e a braçadeira.

As recomendações de 21 a 23 não serão mencionadas neste artigo, pois trata de questões específicas de contaminação por C. difficile.

\section{Recomendação para limpeza/desinfecção dos cabos de ECG, oximetro e termômetros eletrônicos}

24: Os cabos devem ser desinfetados por fricção, com Álcool Etílico a 70\%, durante 10 segundos, no mínimo, utilizando diversas gazes, ou algodão embebido em álcool. Se, possível, completar 10 minutos no tempo de exposição.

25: Aguardar, secar naturalmente e armazenar em ambiente limpo e seco.

O grau de concordância entre os experts, para essas recomendações, obteve média igual a 1 , desta forma, não sofreu alteração.

No entanto, uma das referências apontadas por um dos experts, que trata de um protocolo operacional padrão do serviço de atendimento médico de urgência (SAMU), de Franca - São Paulo ${ }^{(19)}$ traz como informação que, para a limpeza dos cabos de oxímetro, conforme recomendação do fabricante pode-se utilizar panos embebidos em sabão e água e para retirada do sabão, deveria ser passado um pano embebido, apenas, em água.

Assim sendo, entendendo a necessidade de realizar a limpeza antes da desinfecção, acrescentam-se as seguintes recomendações:

- Para limpeza dos cabos de ECG, oxímetro e termômetros eletrônicos, deve ser utilizado um pano com água e detergente neutro passar em toda a superfície do dispositivo;

- Depois retirar todo o detergente com pano úmido embebido em água; 
- Após a limpeza o dispositivo deverá ser seco com pano seco e limpo;

26: Os cabos devem ser desinfetados por fricção, com solução a base de quaternário de amônia, durante 5 segundos no, mínimo, ou conforme orientação do fabricante quanto ao tempo de exposição, utilizando diversas gazes ou algodão embebidos na solução.

27: Passar um pano limpo e úmido (embebido em água) para retirada do excesso do produto. 0 pano não deve estar encharcado para não danificar o equipamento eletrônico.

Referente à utilização do quaternário de amônia como desinfetante de escolha para estes dispositivos, houve um grau de concordância de 0,73 . Porém, a maioria dos experts preferiu não justificar, desta forma, tal recomendação foi analisada com cuidado, revendo todos os estudos selecionados e as referências encontradas sobre o uso deste produto.

Assim sendo, optou-se por descartar as recomendações 26 e 27 e manter, apenas, a opção de desinfecção com álcool etílico, uma vez que, o álcool é mais eficaz comparado ao quaternário de amônia, além, de ser um produto de fácil acesso, baixo custo, e não haver qualquer limitação quanto ao seu uso que justifique a necessidade de existir outra opção para desinfetar estes dispositivos.

28: Estes equipamentos devem ser limpos e desinfetados, sempre que visivelmente sujos, a cada troca de turno e após a alta do paciente.

A limpeza e desinfecção destes equipamentos não sofrem qualquer alteração quanto a sua freqüência.

Porém, observando tudo que foi apontado pelos experts quanto à desinfecção das braçadeiras de esfigmomanômetro, acrescenta-se a desinfecção com álcool etílico a 70\%, sem limpeza prévia, a cada uso entre pacientes distintos.

Todas as alterações nas recomendações, sobre 0 processo de limpeza e desinfecção dos dispositivos de monitoramento não crítico construídas pelos autores, ocorreram a partir da avaliação dos experts, desta forma obteve-se uma nova maneira de realizar estes processos de trabalho e estes foram escritos em formato de protocolo dando origem ao produto final da pesquisa original.

\section{CONCLUSÃO}

Este estudo discutiu os processos de limpeza e desinfecção dos principais dispositivos de monitoramento não críticos, e a participação destes como veículos de infecção cruzada e, traz, ainda, uma proposta de solução para o problema de pesquisa identificado na prática assistencial.

Constatou-se, nos estudos selecionados na revisão integrativa, que os principais dispositivos de monitoramento não críticos (termômetros, esfigmomanômetro, cabos de ECG e oxímetro), podem representar uma ameaça à segurança dos pacientes, sendo uma via de transmissão para as IRAS.
Desta forma, a fim de padronizar os processos de limpeza e desinfecção destes dispositivos, bem como preencher as lacunas do conhecimento teórico/prático identificadas sobre a temática e diminuir os riscos de transmissão de infecção por estes artigos não críticos, foi proposto um protocolo de limpeza e desinfecção dos principais dispositivos de monitoramento não críticos, baseado nas evidências científicas e nas boas práticas.

Este instrumento foi chancelado por um grupo de experts, que fizeram sugestões e críticas que foram consideradas e confrontadas com o conhecimento científico, ocasionando alterações pontuais no protocolo proposto, assim, obtevese o protocolo "final", que é o produto desta pesquisa.

A construção deste instrumento visa orientar a prática baseada nos achados na literatura, considerando, o conhecimento teórico e prático dos experts, no entanto, se faz necessário, que estes procedimentos sejam testados implementando-se o protocolo proposto em futuras pesquisas.

Além dos dispositivos não críticos abordados nesta pesquisa, existem outros equipamentos não invasivos no ambiente de saúde que merecem maior atenção, no que diz respeito a sua participação na ocorrência das IRAS, uma vez que, muitos estudos apontam estes artigos como veículos, ou até mesmo, fontes de bactéria patogênicas.

Dentre estes dispositivos, destacam-se os colchões, brinquedos (utilizados pelas crianças no ambiente de saúde), bombas infusoras, pranchas longas, lâminas e cabos de laringoscopia. Nesse sentido, esta pesquisa apresenta a proposta de um protocolo de limpeza/desinfecção para a Enfermagem que pode ser implantado em diversos serviços e, assim, gerar outros estudos que se proponham a descrever e padronizar a prática de limpeza e desinfecção dos demais dispositivos de saúde não críticos.

\section{REFERÊNCIAS}

1. Ministério da Saúde (BR), Agência Nacional de Vigilância Sanitária. Infecções de Corrente Sanguínea. Orientações para Prevenção de Infecção Primária de Corrente Sanguínea. Brasília: Ministério da Saúde; 2010. 53p.

2. Fernandes AT. Infecção hospitalar e suas interfaces na área da saúde. São Paulo: Atheneu; 2000.

3. Parer $S$, Lotthé $A$, Chardon $P$, Poncet $R$, Jean-Pierre $H$, Jumas-Bilak E. An outbreak oh heterogenous glycopeptideintermediate Stapfylococcus aureus related to a device source in an intensive care unit. Infect Control Hosp Epidemiol. [Internet]. 2012 [Acesso em 13 de Agosto de 2013]; 33(2):167-74. Disponível em: http://www.jstor.org/ stable/10.1086/663703?_redirected.

4. Dijk YV, Bik EM, Hochstenbach-Vernooij S, Vlist GJVD, Savelkoul $\mathrm{PHM}$, Kaan JA et al. Management of an outbreak of enterobacter cloacae in a neonatal unit using simple preventive measures. J Hosp Infect. [Internet]. 2002 [Acesso em 13 de Setembro de 2013]; 51(1): 21-6. Disponível em: http//download. journals.elsevierhealth.com/pdfs/journals/0195-6701/ PIIS0195670102911862.pdf doi: 10.1053/jhin.2002.1186. 
5. Walker N, Gupta R, Cheesbrough J. Blood pressure cuffs: friend or foe? J Hosp Infect. [Internet]. 2006 [Acesso em 15 de Agosto de 2013]; 63(2):167-9. Disponível em: http://www. journalofhospitalinfection.com/article/S0195-6701.

6. Ministério da Saúde (BR), Agência Nacional de Vigilância Sanitária. Segurança do paciente em serviços de saúde. Limpeza e desinfecção de superfícies. Brasília: Ministério da Saúde; 2010.120p.

7. Ministério da Saúde (BR), Agência Nacional de Vigilância Sanitária. Investigação e controle de bactérias multirresistentes. Brasília: Ministério da Saúde; 2007. 21p.

8. Oliveira AC, Paula AO. Infecções relacionadas ao cuidar em saúde no contexto da segurança do paciente: passado, presente e futuro. Rev. Min Enferm. 2013 [Acesso em 20 de Agosto de 2016]; 17(1): 216-220. Disponível em: http://www. reme.org.br/sumario/37.

9. Cullum N, Ciliska D, Haynes RB, Marks S. Enfermagem baseada em evidências. Porto Alegre: Artmed; 2010.

10. Mendes KDS, Silveira RCCP, Galvão CM. Revisão integrativa: método de pesquisa para a incorporação de evidencias na saúde e na enfermagem. Texto \& contexto enferm. [Internet]. 2008; [Acesso em 20 de Outubro 2013]; 17(4): 758-64. Disponível em: http://www.scielo.br/scielo.php?script=sci_ arttext\&pid=S0104-07072008000400018.

11. Souza MT, Silva MD, Carvalho R. Revisão Integrativa: 0 que é e como fazer. Einstein [Internet]. 2010 jun. [Acesso em 22 de Outubro de 2013]; 8(1): 102-6. Disponível em: http://apps. einstein.br/revista/arquivos/PDF/1134-Einsteinv8n1_p102106_port.pdf.

12. Alves KYA, Salvador PTCO, Tourinho FSV, Santos VEP. Análise do conceito "Protocolo de enfermagem" a partir da visão evolucionária de Rodgers.J Nurs UFPE on line. [Internet]. 2014 Jan. [Acesso em 6 de Janeiro de 2014]; 8(1):177-82. Disponível em: http://www.revista.ufpe.br/revistaenfermagem/index. php/revista/article/view/4680 doi: 10.5205/reuol.484339594-1-SM.0801201425.

13. Ferhing RJ. The Fehring model. In: Carrol-Johnson P. Classification of Nursing Diagnosis: proceedings of the tenth conference of North American Nursing Diagnoses Association. Phildelphia: Lippincott; 1994. p. 57-9.

14. Neves, RPS . Dispositivos de monitoramento não críticos: aliados ou inimigos? Construindo um protocolo de limpeza/ desinfecção para enfermagem[dissertação]. Niterói: Escola de Enfermagem Aurora de Afonso Costa/Universidade Federal Fluminense; 2014.

15. Baldin N, Munhoz EMB. Snowball (bola de neve): uma técnica metodológica para pesquisa em educação ambiental comunitária. X Congresso Nacional de educação/l seminário Internacional de representações sociais, subjetividade e educação[Intenet]. 2011 [Acesso em 12 de Janeiro de 2014]. Disponível em: http://educere.bruc.com.br/CD2011/ pdf/4398_2342.pdf.

16. Werneck MAF, Faria HP, Campos KFC. Protocolos de cuidados à saúde e de organização do serviço. Núcleo de Educação em Saúde Coletiva - Nescon da Faculdade de Medicina da Universidade Federal de Minas Gerais, Belo Horizonte: Copmed[Internet]. 2009. [Acesso em 15 de Fevereiro de 2014]. Disponível em: https://www.nescon.medicina.ufmg. br/biblioteca/imagem/1750.pdf.
17. Secretaria de Estado de Saúde e Defesa Civil do Rio de Janeiro, Assessoria de Qualidade. Normas para elaboração, controle e revisão de instrumentos normativos. Rio de Janeiro: SEDESC; 2008.15p.

18. Ministério da Saúde (Brasil), Agência Nacional de Vigilância Sanitária. Investigação e controle de bactérias multirresistentes. Brasília: Ministério da Saúde; 2007. 21p.

19. Secretaria Municipal de Saúde de Franca - Serviço de atendimento móvel de urgência. Protocolo operacional Padrão: Biossegurança, limpeza, desinfecção e esterilização. São Paulo; 2012. 